\title{
Magnetized Reissner-Nordstrom-Taub-NUT spacetime and microscopic entropy
}

\author{
Haryanto M. Siahaan ${ }^{\mathrm{a}}(\mathbb{D}$ \\ Department of Physics, Center for Theoretical Physics, Parahyangan Catholic University, Jalan Ciumbuleuit 94, Bandung 40141, Indonesia
}

Received: 25 March 2021 / Accepted: 10 September 2021 / Published online: 21 September 2021

(C) The Author(s) 2021

\begin{abstract}
We present a novel solution describing magnetized spacetime outside an electrically charged massive object equipped with NUT parameter. To get the solution, we employ the Ernst magnetization to the Reissner-NordstromTaub-NUT spacetime as the seed. After discussing some physical aspects of the spacetime, we show that the extremal entropy of a magnetized Reissner-Nordstrom-Taub-NUT black hole can be reproduced by using the Cardy formula.
\end{abstract}

\section{Introduction}

Reissner-Nordstrom black hole is an exact solution in the Einstein-Maxwell theory describing the spacetime outside a collapsing object with an electric charge. Despite it is very unlikely for a collapsing matter to maintain a significant amount of electric charge in the real world, the ReissnerNordstrom solution has been one of the most studied object in gravitational researches [1] especially related to black holes [2-5]. Moreover, inspired by the Kerr/CFT correspondence, the charged black hole/CFT holography has been investigated [6-8] where some properties of extremal or near extremal Reissner-Nordstrom black holes can be reproduced by using some two-dimensional conformal field theory approach.

In addition to the black hole with electric charge, there also exist exact solutions describing a black hole immersed in an external magnetic field. A solution where the magnetic field is considered as some perturbations in the spacetime was introduced by Wald [9], and for the case of a strong magnetic field was proposed by Ernst [10]. In the proposal by Ernst, the magnetized spacetime is obtained by using a Harrison type of transformation applied to a seed solution in EinsteinMaxwell theory. For example, one can use the Kerr-Newman solution as the seed to obtain the magnetized Kerr-Newman

a e-mail: haryanto.siahaan@unpar.ac.id (corresponding author) spacetime [10]. Setting the mass, electric charge, and rotation parameters in the magnetized Kerr-Newman solution yields the Melvin magnetic universe [11]. Interestingly, the presence of an external magnetic field does not change the area of the embedded black hole, but rather deforms the surface as reported in [12]. Various studies on black holes in external magnetic field can be found in literature [13-29]. Some of these studies are motivated by the possibility of strong magnetic field around a black hole due to the presence of rotating hot plasma in its surrounding.

Kerr-Newman-Taub-NUT (KNTN) black hole of EinsteinMaxwell theory is parameterized by the NUT parameter $l$, in addition to the well-known other black hole parameters, namely mass $m$, rotation $a$, and electric charge $q$ [1]. The Taub-NUT solution is a special case of this KNTN family, obtained after setting the rotational parameter $a$ and electric charge $q$ to vanish. Despite the obscure realization of the NUT parameter in our real world, spacetime solutions with NUT charge have been used to extend our understanding of gravity in many ways. For example, the motion of charged test particle was studied in [30], the area product and mass formula is investigated in [31], and the gravitomagnetism in this spacetime is reported in [32]. Furthermore, solution with NUT parameter can also exist in gravitational theories beyond Einstein-Maxwell, for example in scalar-tensor [33], Randall-Sundrum braneworld [34], and low energy heterotic string [35] theories. In a recent work [36], the authors show that the Misner string contribution to the Taub-NUTAdS entropy can be renormalized by introducing the GaussBonnet term.

In this paper we consider the $a=0$ limit of KNTN family, namely the Reissner-Nordstrom-Taub-NUT (RNTN) solution, as the seed to be magnetized. The magnetized ReissnerNordstrom spacetime had been reported decades ago [12] where the author used Reissner-Nordstrom solution as the seed, and the associated Kerr/CFT correspondence study was carried out in [37]. The significance of magnetized Reissner- 
Nordstrom-Taub-NUT (MRNTN) solution at the moment is more theoretical rather than practical to our understanding of black holes. The linear approximation introduced by Wald [9] to model a black hole immersed by the magnetic field is found to be sufficient in describing the black hole and magnetic field interaction. Nevertheless, one would like to know how our present black hole thermodynamical relations can extend to the case of the charged black hole with NUT parameter immersed in a fully non-linear external magnetic field. These are interesting since the black hole mechanics for magnetized spacetime and spacetime with NUT parameter are still under development [19,38-40].

To get the magnetized solution, we employ the Ernst magnetization transformation to the RNTN spacetime. Our study differs from the work by Frolov et al. [41] in the sense that if all parameters in the solution except magnetic field parameter are switched off, we get the Melvin magnetic universe [11]. After establishing the magnetized solution, aspects of the spacetime such as Hawking temperature and thermodynamics are discussed. We then test the conjectured extremal Kerr/CFT correspondence for the new spacetime solution, where we can recover the extremal entropy by using the asymptotic symmetry group method [42-44].

In fact, this Kerr/CFT correspondence conjecture has been applied to black hole case with NUT parameter [45,46], and to magnetized black holes in the absence of NUT parameter $[37,47,48]$. It motivates us to investigate the Kerr/CFT extension to the case of magnetized Reissner-Nordstrom-TaubNUT spacetime solution introduced in this paper. In fact, the $U(1) \times S L(2, R)$ symmetric form of the near horizon geometry of extremal magnetized Reissner-Nordstrom-TaubNUT spacetime showed in Sect. 4 confirms the possibility to extend Kerr/CFT holography to the case of magnetized Reissner-Nordstrom-Taub-NUT solution [43]. Despite the fact where it seems unlikely we can find some astrophysical process that requires the new spacetime solution presented in this paper, for example, massive charged object with NUT charge immersed in a strong magnetic field, the Kerr/CFT correspondence investigation for this spacetime can test how general the correspondence can extent.

The organization of this paper is as follows. In the next section, we construct the MRNTN solution by employing the Ernst magnetization to the RNTN spacetime as the seed. The area, entropy, Hawking temperature, and thermodynamics of the black hole in the spacetime are discussed in Sect. 3. In Sect. 4, we compute the entropy of extremal MRNTN black hole using microscopic formula. Finally, we give conclusions and discussions. In this paper, we consider the natural units $c=\hbar=k_{B}=G_{4}=1$.

\section{Construction of magnetized Reissner-Nordstrom-Taub-NUT spacetime}

\subsection{Ernst magnetization}

RNTN spacetime is an exact solution of Einstein-Maxwell equations,

$R_{\mu \nu}=2 F_{\mu \alpha} F_{v}^{\alpha}-\frac{1}{2} g_{\mu \nu} F_{\alpha \beta} F^{\alpha \beta}$,

where $F_{\mu \nu}=\partial_{\mu} A_{v}-\partial_{\nu} A_{\mu}$ is the field strength tensor. Unlike the Reissner-Nordstrom solution as its null NUT counterpart, RNTN spacetime lacks of the asymptotic flatness due to the presence of NUT parameter. Nevertheless, many aspects of RNTN spacetime have been studied in literature, including its rotating generalization known as the Kerr-Newman-Taub-NUT solution. The tunneling method calculation for Hawking temperature from the black hole was performed in $[49,50]$, the thermodynamics studies are presented in [38-40,51], area and Smarr formulas are discussed in $[31,52]$, the motion of test particle in this spacetime are studied in [30,53], and the possibility of destroying a RNTN black hole is investigated in [2]. However, RNTN spacetime solution studied in these works has not incorporated some non-perturbative external magnetic fields ${ }^{1}$ as considered by Ernst and Wild in [10,12] for Reissner-Nordstrom and Kerr black holes. This section is dedicated to construct a magnetized version of RNTN spacetime by following the Ernst method [10]. As we have mentioned before that the magnetized Reissner-Nordstrom solution has been reported in [12], the new spacetime presented in this paper can be considered as the Taub-NUT generalization to that solution.

As a start, let us review the Ernst magnetization prescription which is basically a type of Harrison transformation. We consider the following stationary and axial symmetric line element,

$\mathrm{d} s^{2}=f(\omega \mathrm{d} t-\mathrm{d} \phi)^{2}+f^{-1}\left(e^{2 \gamma} \mathrm{d} \chi \mathrm{d} \bar{\chi}-\rho^{2} \mathrm{~d} t^{2}\right)$,

that is known as the Lewis-Papapetrou-Weyl (LPW) type of metric. In the metric above, the functions $f, \gamma$, and $\omega$ depend on $\chi$, and we have used the -+++ signs convention for the spacetime. Now let us also consider two kind of complex potentials, namely the electromagnetic Ernst potential

$\Phi=A_{\phi}+i \tilde{A}_{\phi}$,

and the gravitational one

$\mathcal{E}=f+\Phi \bar{\Phi}-i \Psi$.

\footnotetext{
${ }^{1}$ Perturbative or weak external magnetic fields in a black hole background was introduced by Wald [9], and has been extended to some other general black hole cases [41].
} 
Note that the real part of the potential $\Phi$ is the $A_{\phi}$ component of the vector potential $A_{\mu}$, and the imaginary part comes from the vector field $\tilde{A}_{\mu}$ which builds the dual field strength tensor $\tilde{F}_{\mu \nu}=\partial_{\mu} \tilde{A}_{\nu}-\partial_{\nu} \tilde{A}_{\mu}$ where $\tilde{F}_{\mu \nu}=\frac{1}{2} \varepsilon_{\mu \nu \alpha \beta} F^{\alpha \beta}$. To find the solution for $A_{t}$ component, we can use

$-i \frac{\rho}{f} \nabla \tilde{A}_{\phi}=\nabla A_{t}+\omega \nabla A_{\phi}$

The twist potential $\Psi$ in gravitational potential (2.4) is related to the metric functions and vector potential by

$-i \nabla \Psi=\frac{f^{2}}{\rho} \nabla \omega+2 \bar{\Phi} \nabla \Phi$.

Using these two potentials above, the Einstein-Maxwell equations (2.1) can be rewritten in the form of wave equations known as the Ernst equations [1],

$$
\begin{aligned}
& (\mathcal{E}+\overline{\mathcal{E}}+\Phi \bar{\Phi}) \nabla^{2} \mathcal{E}=2(\nabla \mathcal{E}+2 \bar{\Phi} \nabla \Phi) \cdot \nabla \mathcal{E} \\
& (\mathcal{E}+\overline{\mathcal{E}}+\Phi \bar{\Phi}) \nabla^{2} \Phi=2(\nabla \mathcal{E}+2 \bar{\Phi} \nabla \Phi) \cdot \nabla \Phi
\end{aligned}
$$

There exist some transformations that leave the two Eqs. (2.7) and (2.8) above to be invariant, for example the Harrison transformation [54]. In [10] Ernst showed that one can perform a magnetization of a known spacetime using a type of Harrison transformation, namely

$\mathcal{E} \rightarrow \mathcal{E}^{\prime}=\Lambda^{-1} \mathcal{E}$ and $\Phi \rightarrow \Phi^{\prime}=\Lambda^{-1}(\Phi-b \mathcal{E})$,

where

$\Lambda=1-2 b \Phi+b^{2} \mathcal{E}$.

Above, the constant $b$ represents the strength of external magnetic field in the spacetime. ${ }^{2}$ The transformation (2.9) leaves Eqs. (2.7) and (2.8) unchanged for the new potentials $\mathcal{E}^{\prime}$ and $\Phi^{\prime}$ from which a new set of solutions $\left\{A_{\mu}^{\prime}, g_{\mu \nu}^{\prime}\right\}$ to the Einstein-Maxwell equations can be extracted.

The magnetization transformation (2.9) acting on the potentials $\mathcal{E}$ and $\Phi$ yields the transformed metric functions in the LPW form (2.2) as the followings

$f^{\prime}=\operatorname{Re}\left\{\mathcal{E}^{\prime}\right\}-\Phi^{\prime} \bar{\Phi}^{\prime}=\frac{f}{|\Lambda|^{2}}$,

and

$\nabla \omega^{\prime}=|\Lambda|^{2} \nabla \omega+\frac{\rho}{f}(\Lambda \nabla \bar{\Lambda}-\bar{\Lambda} \nabla \Lambda)$,

while $\gamma$ remains unchanged. Since all the incorporating functions in the metric (2.2) depend on $\rho$ and $z$ only, one can define the operator $\nabla$ in the flat Euclidean space

$\mathrm{d} \chi \mathrm{d} \bar{\chi}=\mathrm{d} \rho^{2}+\mathrm{d} z^{2}$,

where we have $d \chi=d \rho+i d z$ and $\nabla=\partial_{\rho}+i \partial_{z}$ accordingly.

\footnotetext{
${ }^{2}$ Here we use a quite different label for the strength of magnetic field, where in some other works $[37,47,48]$ is used $B=2 b$ instead.
}

However, the typical spacetime solution in EinsteinMaxwell theory that contains black hole can be expressed in the Boyer-Lindquist type coordinate system $\{t, r, x=\cos \theta$, $\phi$ \}. Therefore, we could bring the LPW line element (2.2) into a Boyer-Lindquist type, where some terms in the line element can be rewritten as

$\mathrm{d} \chi \mathrm{d} \bar{\chi}=\frac{\mathrm{d} r^{2}}{\Delta_{r}}+\frac{\mathrm{d} x^{2}}{\Delta_{x}}$

with $\Delta_{r}=\Delta_{r}(r)$ and $\Delta_{x}=\Delta_{x}(x)$. Accordingly, the corresponding operator $\nabla$ will read $\nabla=\sqrt{\Delta_{r}} \partial_{r}+i \sqrt{\Delta_{x}} \partial_{x}$, and Eq. (2.5) allows us to write

$\partial_{r} A_{t}=\frac{\Delta_{x}}{f} \partial_{x} \tilde{A}_{\phi}-\omega \partial_{r} A_{\phi}$,

and

$$
-\partial_{x} A_{t}=\frac{\Delta_{r}}{f} \partial_{r} \tilde{A}_{\phi}+\omega \partial_{x} A_{\phi},
$$

after using $\rho^{2}=\Delta_{r} \Delta_{x}$. The two Eqs. (2.15) and (2.16) are useful later in obtaining the $A_{t}$ component associated to the magnetized spacetime according to (2.9). To complete some details on magnetization procedure, another subtleties that may be required to obtain the magnetized solution are

$\partial_{r} \omega^{\prime}=|\Lambda|^{2} \partial_{r} \omega-\frac{\Delta_{x}}{f} \operatorname{Im}\left\{\Lambda \partial_{x} \bar{\Lambda}-\bar{\Lambda} \partial_{x} \Lambda\right\}$,

and

$\partial_{x} \omega^{\prime}=|\Lambda|^{2} \partial_{x} \omega+\frac{\Delta_{r}}{f} \operatorname{Im}\left\{\Lambda \partial_{r} \bar{\Lambda}-\bar{\Lambda} \partial_{r} \Lambda\right\}$.

In the next section, this Ernst magnetization procedure will be employed with the RNTN solution as the seed to obtain the MRNTN spacetime.

\subsection{Magnetized Reissner-Nordstrom-Taub-NUT spacetime}

Let us start with the RNTN line element

$$
\begin{aligned}
\mathrm{d} s^{2}= & -\frac{\Delta_{r}(\mathrm{~d} t+2 l x \mathrm{~d} \phi)^{2}}{r^{2}+l^{2}}+\left(r^{2}+l^{2}\right) \Delta_{x} \mathrm{~d} \phi^{2} \\
& +\left(r^{2}+l^{2}\right)\left(\frac{\mathrm{d} r^{2}}{\Delta_{r}{ }^{2}}+\frac{\mathrm{d} x^{2}}{\Delta_{x}{ }^{2}}\right)
\end{aligned}
$$

where $\Delta_{r}=r^{2}-2 m r+q^{2}-l^{2}$ and $\Delta_{x}=1-x^{2}$. Together with the vector potential

$A_{\mu} \mathrm{d} x^{\mu}=\frac{q r(\mathrm{~d} t+2 l x \mathrm{~d} \phi)}{r^{2}+l^{2}}$,

the metric (2.19) solve the Einstein-Maxwell equation (2.1). In getting the magnetized version of RNTN solution above, we need to bring the seed metric (2.19) into the LPW form 
(2.2). It is straightforward to show that the associated metric functions are

$f=\frac{\left(r^{2}+l^{2}\right)^{2}+x^{2}\left(3 l^{4}-r^{4}+l^{2}\left\{8 m r-6 r^{2}-4 q^{2}\right\}\right)}{\left(r^{2}+l^{2}\right)}$,

$\omega=\frac{2 l x \Delta_{r}}{\left(r^{2}+l^{2}\right)^{2}+x^{2}\left(3 l^{4}-r^{4}+l^{2}\left\{8 m r-6 r^{2}-4 q^{2}\right\}\right)}$,

$\rho^{2}=\Delta_{x} \Delta_{r}$

and

$e^{2 \gamma} f^{-1}=r^{2}+l^{2}$.

By using these functions and vector solution (2.20), one can find the corresponding gravitational potential

$\bar{f}_{1}=-8 b q x r\left(1-b^{2} x^{2} q^{2}-2 b^{2} r^{2} x^{2}+3 b^{2} m r x^{2}\right)$,

$\bar{f}_{2}=1+b^{2} x^{2} q^{2}\left(9 b^{2} x^{2} q^{2}-2\right)+r^{4} b^{4}\left(15 x^{4}+7-6 x^{2}\right)$ $-8 r^{3} b^{4} m x^{2}\left(5 x^{2}+1\right)$ $+4 r^{2} b^{2}\left(3 b^{2} x^{2} q^{2}+1-3 x^{2}+3 b^{2} x^{4} q^{2}+9 b^{2} m^{2} x^{4}\right)$ $-16 r b^{2} m x^{2}\left(2 b^{2} x^{2} q^{2}-1\right)$,

$\bar{f}_{3}=-8 b^{3} q x\left(m x^{2}+2 r\right)$,

$\bar{f}_{4}=b^{2}\left[4 b^{2} m^{2} x^{4}+24 b^{2} r x^{2} \Delta_{x} m-9 b^{2} x^{4}\left(r^{2}+2 q^{2}\right)\right.$ $\left.-6 x^{2}\left(q 2 b^{2}+5 b^{2} r^{2}-1\right)+7 b^{2} r^{2}+2\right]$,

$\bar{f}_{5}=0$,

$\bar{f}_{6}=b^{4}\left(1+3 x^{2}\right)^{2}$,

and

$\omega^{\prime}=\frac{\sum_{k=0}^{5} \bar{\omega}_{k} x^{k}}{\left(r^{2}+l^{2}\right)^{2}+x^{2}\left(3 l^{4}-r^{4}+l^{2}\left\{8 m r-6 r^{2}-4 q^{2}\right\}\right)}$

$\mathcal{E}=\frac{l^{3}\left(1+3 x^{2}\right)+i l^{2}\left(3 r \Delta_{x}+2 m x^{2}\right)+l\left(6 m r x^{2}-3 x^{2}\left\{r^{2}+q^{2}\right\}-r^{2}\right)+i r\left(q^{2} x^{2}+r^{2} \Delta_{x}\right)}{l+i r}$,

and the electromagnetic one

$\Phi=\frac{-i q x(i r-l)}{i r+l}$.

Furthermore, from (2.10), these two potentials provide us

$$
\begin{aligned}
\Lambda= & 1+\frac{i 2 b q x(i r-l)}{i r+l} \\
& +\frac{b^{2}}{i r+l}\left\{l^{3}\left(1+3 x^{2}\right)+i l^{2}\left(3 r \Delta_{x}+2 m x^{2}\right)\right. \\
& \left.+l\left(6 m r x^{2}-3 x^{2}\left\{r^{2}+q^{2}\right\}-r^{2}\right)+i r\left(q^{2} x^{2}+r^{2} \Delta_{x}\right)\right\} .
\end{aligned}
$$

Finally, we can get the magnetized Ernst potentials $\mathcal{E}^{\prime}$ and $\Phi^{\prime}$ after inserting (2.25), (2.26), and (2.27) into (2.9). The resulting magnetized line element can be written as

$$
\begin{aligned}
\mathrm{d} s^{2}= & \frac{1}{f^{\prime}}\left\{-\rho^{2} \mathrm{~d} t^{2}+e^{2 \gamma}\left(\frac{\mathrm{d} r^{2}}{\Delta_{r}}+\frac{\mathrm{d} x^{2}}{\Delta_{x}}\right)\right\} \\
& +f^{\prime}\left(\mathrm{d} \phi-\omega^{\prime} \mathrm{d} t\right)^{2},
\end{aligned}
$$

where the functions appearing in metric above read

$$
f^{\prime}=\frac{\left\{\left(r^{2}+l^{2}\right)^{2}+x^{2}\left(3 l^{4}-r^{4}+l^{2}\left\{8 m r-6 r^{2}-4 q^{2}\right\}\right)\right\}}{\sum_{k=0}^{6} \bar{f}_{k} l^{k}},
$$

where

$$
\begin{aligned}
\bar{f}_{0}= & r^{2}\left[b^{4} r^{4} \Delta_{x}^{2}+2 b^{2} r^{2} \Delta_{x}\left(1+b^{2} q^{2} x^{2}\right)\right. \\
& \left.+1+b^{2} q^{2} x^{2}\left(6+b^{2} q^{2} x^{2}\right)\right]
\end{aligned}
$$

where

$$
\begin{aligned}
\bar{\omega}_{5}= & b^{4} l\left(4 l^{2} m^{2}+4 r^{3} m-4 l^{2} m r-r^{4}+6 r^{2} l^{2}\right. \\
& \left.-4 q^{2} r^{2}-4 q^{2} l^{2}+q^{4}+3 l^{4}\right) \Delta_{r}, \\
\bar{\omega}_{4}= & -2 b^{3} q\left(4 l^{2} m+r^{3}-3 r l^{2}\right) \Delta_{r}, \\
\bar{\omega}_{3}= & -2 b^{2} l\left(b^{2} l^{4}-2 b^{2} l^{2} q^{2}+6 b^{2} l^{2} r^{2}-2 b^{2} l^{2} m r\right. \\
& \left.-2 b^{2} q^{2} r^{2}-3 q^{2}+b^{2} r^{4}+2 b^{2} r^{3} m\right) \Delta_{r}, \\
\bar{\omega}_{2}= & 2 b q r\left(b^{2} q^{2} r^{2}-3 b^{2} l^{2} q^{2}-2 b^{2} r^{3} m-4 b^{2} l^{2} r^{2}\right. \\
& \left.+r^{2}+6 b^{2} l^{2} m r+4 b^{2} l^{4}+l^{2}\right), \\
\bar{\omega}_{1}= & -l\left(b^{4} l^{4}-6 l^{2} b^{4} r^{2}-1-3 b^{4} r^{4}\right) \Delta_{r}, \\
\bar{\omega}_{0}= & -2 r q b\left(r^{2}+l^{2}\right)\left(b^{2} l^{2}+1-b^{2} r^{2}\right) .
\end{aligned}
$$

Furthermore, the accompanying vector solution to this magnetized metric can be expressed in the following compact form

$$
A_{\mu}^{\prime} \mathrm{d} x^{\mu}=\frac{\mathrm{d} t \sum_{k=0}^{6} \bar{c}_{k} x^{k}+\mathrm{d} \phi \sum_{k=0}^{4} \bar{d}_{k} x^{k}}{\sum_{k=0}^{4} \bar{f}_{k} x^{k}} .
$$

The functions $\bar{c}_{k}$ and $\bar{d}_{k}$ appearing in the numerator of r.h.s. in Eq. (2.31) are

$$
\begin{aligned}
\bar{c}_{6}= & b^{6} q\left\{q^{4} r-\left(2 r^{3}-8 l^{2} m+10 r l^{2}\right) q^{2}-12 r l^{2} m^{2}\right. \\
& \left.+16 r^{2} l^{2} m-2 r^{3} l^{2}-8 l^{4} m+r^{5}+9 r l^{4}\right\} \Delta_{r}, \\
\bar{c}_{5}= & -2 b^{5} l\left\{7 q^{4}+\left(2 r^{2}-12 m r-10 l^{2}\right) q^{2}+4 l^{2} m^{2}\right. \\
& \left.+4 m r^{3}+6 r^{2} l^{2}-4 l^{2} r m-r^{4}+3 l^{4}\right\} \Delta_{r}, \\
\bar{c}_{4}= & q b^{4}\left\{\left(b^{2} r+8 b^{2} m\right) l^{6}+\left(3 r+5 b^{2} r^{3}+4 b^{2} r^{2} m\right.\right. \\
& \left.-8 q^{2} b^{2} m+12 b^{2} r m^{2}-10 q^{2} b^{2} r-8 m\right) l^{4} \\
& +\left(\left(-5 r^{5}+4 r^{3} m^{2}-4 q^{2} r^{3}+16 m r^{4}-20 q^{2} r^{2} m\right.\right. \\
& \left.\left.+9 q^{4} r\right) b^{2}+44 m r^{2}-26 r^{3}-28 r m^{2}+6 q^{2} r+8 q^{2} m\right) l^{2}
\end{aligned}
$$




$$
\begin{aligned}
& -r^{5}+3 q^{4} b^{2} r^{3}+12 q^{2} r^{2} m-4 m r^{4}-2 q^{2} b^{2} r^{5}-9 q^{4} r \\
& \left.+4 b^{2} m r^{6}+2 q^{2} r^{3}-b^{2} r^{7}-4 q^{2} b^{2} m r^{4}\right\}, \\
\bar{c}_{3}= & -4 b^{3} l\left\{\left(1-b^{2} l^{2}+5 b^{2} r^{2}\right) q^{4}\right. \\
& +\left(4 r b^{2} l^{2} m-8 m r+4 b^{2} r^{4}-12 r^{3} b^{2} m+6 r^{2}\right. \\
& \left.-12 b^{2} l^{2} r^{2}-2 l^{2}\right) q^{2}+\left(4 r^{4} m^{2}-r^{6}-4 l^{2} r^{2} m^{2}\right. \\
& \left.+16 l^{2} r^{3} m-5 l^{2} r^{4}+l^{6}+5 l^{4} r^{2}\right) b^{2}+r^{4}-2 r^{2} l^{2} \\
& \left.+l^{4}+4 r^{2} m^{2}+4 l^{2} r m-4 m r^{3}\right\}, \\
\bar{c}_{2}= & b^{2} q r\left\{\left(3 l^{2} r^{4}-20 l^{2} r^{3} m-11 l^{4} r^{2}+3 q^{2} r^{4}+14 l^{2} r^{2} q^{2}\right.\right. \\
& \left.-9 l^{4} q^{2}+6 l^{4} r m+9 l^{6}-r^{6}-2 m r^{5}\right) b^{4} \\
& +\left(44 r^{2} l^{2}-14 q^{2} r^{2}+6 l^{2} q^{2}-2 l^{4}+6 r^{4}\right. \\
& \left.\left.+4 m r^{3}-36 l^{2} r m\right) b^{2}-9 q^{2}-r^{2}+6 m r+5 l^{2}\right\}, \\
\bar{c}_{1}= & -2 b l\left\{\left(6 l^{2} r^{2} q^{2}+3 q^{2} r^{4}-l^{4} q^{2}+3 l^{2} r^{4}-7 l^{4} r^{2}\right.\right. \\
& \left.+3 r^{6}-6 m r^{5}+l^{6}+2 l^{4} r m-12 l^{2} r^{3} m\right) b^{4} \\
& +\left(4 l^{2} r m-4 m r^{3}-4 r^{2} l^{2}-6 q^{2} r^{2}-2 l^{2} q^{2}+2 r^{4}\right. \\
& \left.\left.+2 l^{4}\right) b^{2}+2 m r+l^{2}-q^{2}-r^{2}\right\}, \\
\bar{c}_{0}= & 9 r\left(1-5 b^{6} r^{2} l^{4}-5 b^{4} r^{4}+5 b^{6} r^{4} l^{2}-18 b^{4} r^{2} l^{2}\right. \\
& \left.-l^{6} b^{6}+b^{6} r^{6}+b^{2} l^{2}-5 b^{2} r^{2}-l^{4} b^{4}\right),
\end{aligned}
$$

and

$$
\begin{aligned}
\bar{d}_{4}= & -b^{3}\left\{\left(12 q^{2} r^{2}-32 m r q^{2}-40 m r^{3}\right.\right. \\
& \left.+36 r^{2} m^{2}+15 r^{4}+9 q^{4}\right) l^{2}+r^{2}\left(q^{2}-r^{2}\right)^{2} 9 l^{6} \\
& \left.+\left(4 m^{2}-18 q^{2}+24 m r-9 r^{2}\right) l^{4}\right\}, \\
\bar{d}_{3}= & 6 b^{2} l q\left\{3 m r^{2}-q^{2} r+l^{2} m-2 r^{3}\right\}, \\
\bar{d}_{2}= & b\left\{\left(-12 l^{2} r^{2} q^{2}+6 l^{2} r^{4}+2 r^{6}\right.\right. \\
& \left.+8 l^{2} r^{3} m-2 q^{2} r^{4}+6 l^{4} q^{2}-6 l^{6}-24 l^{4} r m+30 l^{4} r^{2}\right) b^{2} \\
& \left.+r^{4}+l^{2} q^{2}-8 l^{2} r m+6 r^{2} l^{2}-3 q^{2} r^{2}-3 l^{4}\right\}, \\
\bar{d}_{1}= & 2 q r l\left(1+6 b^{2} l^{2}\right), \\
\bar{d}_{0}= & -b\left(l^{2}+r^{2}\right)\left(b^{2} l^{4}+l^{2}+6 b^{2} l^{2} r^{2}+b^{2} r^{4}+r^{2}\right),
\end{aligned}
$$

while the function $\bar{f}_{k}$ in the denominator are

$$
\begin{aligned}
\bar{f}_{4}= & b^{4}\left\{4 l^{2} m^{2}\left(9 r^{2}+l^{2}\right)+8 r l^{2} m\left(3 l^{2}-5 r^{2}-4 q^{2}\right)\right. \\
& +r^{6}+\left(15 l^{2}-2 q^{2}\right) r^{4}+\left(q^{4}+12 l^{2} q^{2}-9 l^{4}\right) r^{2} \\
& \left.-18 l^{4} q^{2}+9 l^{6}+9 l^{2} q^{4}\right\}, \\
\bar{f}_{3}= & 8 b^{3} l q\left\{q^{2} r-3 m r^{2}-l^{2} m+2 r^{3}\right\}, \\
\bar{f}_{2}= & 2 b^{2}\left\{\left(12 l^{4} r m+3 l^{6}-4 l^{2} r^{3} m+q^{2} r^{4}+6 l^{2} r^{2} q^{2}\right.\right. \\
& \left.-3 l^{2} r^{4}-15 l^{4} r^{2}-3 l^{4} q^{2}-r^{6}\right) b^{2} \\
& \left.-r^{4}+3 q^{2} r^{2}+8 l^{2} r m-6 r^{2} l^{2}+3 l^{4}-l^{2} q^{2}\right\}, \\
\bar{f}_{1}= & -8 b q r l\left\{1+2 b^{2} l^{2}\right\}, \\
\bar{f}_{0}= & \left(l^{2}+r^{2}\right)\left\{l^{4} b^{4}+6 b^{4} r^{2} l^{2}+2 b^{2} l^{2}+2 b^{2} r^{2}+b^{4} r^{4}+1\right\} .
\end{aligned}
$$

The solutions (2.28) and (2.31), which will be referred as the magnetized Reissner-Nordstrom-Taub-NUT (MRNTN) solution, can be considered as the Taub-NUT extension of magnetized Reissner-Nordstrom black holes proposed in [10]. For a black hole immersed in the magnetic field, the area of its horizon is the same to that of the non-magnetized one $[37,47,48]$. As one would expect, the same result appears here where the radii of MRNTN black hole is that of the RNTN horizons, namely $r_{ \pm}=m \pm \sqrt{m^{2}-q^{2}+l^{2}}$.
Accordingly, the extremal conditions for the magnetized and non-magnetized RNTN black hole also coincide, namely $m^{2}+l^{2}=q^{2}$.

\section{Some aspects of the spacetime}

\subsection{Kretschmann scalar}

In studying the curved spacetime, Kretschmann scalar $K=$ $R_{\alpha \beta \mu \nu} R^{\alpha \beta \mu \nu}$ can be used to identify the existence of true singularity at origin in the spacetime. It is well known that a spacetime with NUT parameter is regular at the origin, but suffers the conic singularity where the periodicity of $\phi$ coordinate is no longer $2 \pi$. Note that the conic singularity in spacetime coming from the presence of NUT parameter differs to that as the result of external magnetic field. The latter case can be cured by performing a scaling in $\phi$ coordinate [14].

Obviously, the full expression for Kretschmann scalar of MRNTN spacetime is lengthy even at $x=0$. Nevertheless, the regularity of this quantity at origin can be identified by looking at its denominator, where the Kretschmann scalar can be expressed as

$$
K \sim \frac{1}{\left(\sum_{k=0}^{6} c_{k} l^{k}\right)^{6}},
$$

with

$$
\begin{aligned}
c_{6}= & b^{4}\left(3 x^{2}+1\right)^{2}, \\
c_{5}= & 0, \\
c_{4}= & b^{2}\left(4 b^{2} m^{2} x^{4}+24 b^{2} m r x^{4}-18 x^{4} q^{2} b^{2}-9 b^{2} r^{2} x^{4}\right. \\
& \left.+24 b^{2} m r x^{2}-6 b^{2} q^{2} x^{2}-30 b^{2} r^{2} x^{2}+7 b^{2} r^{2}+6 x^{2}+2\right), \\
c_{3}= & -8 b^{3} q x\left(m x^{2}+2 r\right), \\
c_{2}= & 36 b^{4} m^{2} r^{2} x^{4}-32 b^{4} m q^{2} r x^{4}-40 b^{4} m r^{3} x^{4}+9 b^{4} q^{4} x^{4} \\
& +12 r^{2} b^{4} q^{2} x^{4}+15 r^{4} b^{4} x^{4}-8 b^{4} m r^{3} x^{2} \\
& +12 r^{2} b^{4} q^{2} x^{2}-6 r^{4} b^{4} x^{2}+7 r^{4} b^{4} \\
& +16 b^{2} m r x^{2}-2 b^{2} q^{2} x^{2}-12 b^{2} r^{2} x^{2}+4 b^{2} r^{2}+1, \\
c_{1}= & -8 b q r x\left(3 b^{2} m r x^{2}-b^{2} q^{2} x^{2}-2 b^{2} r^{2} x^{2}+1\right), \\
c_{0}= & r^{2}\left(b^{4} q^{4} x^{4}-2 r^{2} b^{4} q^{2} x^{4}+r^{4} b^{4} x^{4}\right. \\
& +2 r^{2} b^{4} q^{2} x^{2}-2 r^{4} b^{4} x^{2}+r^{4} b^{4}+6 b^{2} q^{2} x^{2}-2 b^{2} r^{2} x^{2} \\
& \left.+2 b^{2} r^{2}+1\right) .
\end{aligned}
$$

So it is easy to notice that this quantity is not singular for $r=$ 0 and non-vanishing $l$. Moreover, after setting the external magnetic field parameter $b$ and electric charge $q$ vanish in Eq. (3.1), the Kretschmann scalar for Taub-NUT spacetime can be recovered, i.e.

$K=\frac{48}{\left(r^{2}+l^{2}\right)^{6}}\left\{\left(l^{4}-m l^{3}+3 l^{3} r+3 l^{2} m r-3 l^{2} r^{2}\right.\right.$ 


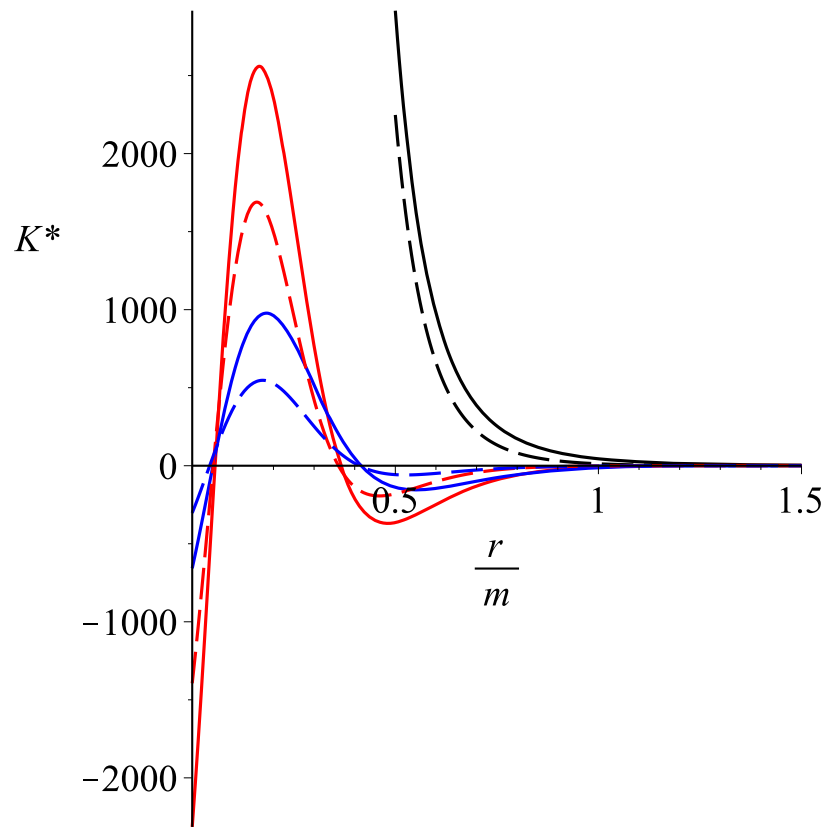

Fig. 1 Plots of the dimensionless Kretschmann scalar, i.e. $K^{*}=$ $m^{4} R_{\alpha \beta \mu \nu} R^{\alpha \beta \mu \nu}$, for $q=0.1$. The case of $b m=0.1$ are represented by the solid lines, and $b m=0.5$ by the dashed lines. The black lines are the cases of $l=0$, red lines are $l=0.5$, and blue lines are $l=0.6$

$$
\begin{aligned}
& \left.+3 m r^{2} l-r^{3} l-m r^{3}\right) \times\left(l^{4}+m l^{3}-3 l^{3} r\right. \\
& \left.\left.+3 l^{2} m r-3 l^{2} r^{2}-3 m r^{2} l+r^{3} l-m r^{3}\right)\right\} .
\end{aligned}
$$

To proceed with some numerical studies, let us consider the quantity (3.1) on equator where its full expression is given in the appendix. Some results are presented in Fig. 1 where we find that the typical behavior of spacetime with NUT parameter appear, i.e. the corresponding Kretschmann scalar at origin is regular or finite. As comparison, we also provide the null case of NUT parameter, where the singularity at origin still exist in the spacetime with external magnetic field.

\subsection{Electric charge and angular momentum}

Indeed, as a generalization of the Reissner-Nordstrom spacetime solution, there is an electric charge associated to the MRNTN spacetime. Following [20], the corresponding electric charge can be found as

$$
Q=\frac{|\Lambda(x=1)|^{2}}{2}\left[\tilde{A}_{\phi}\right]_{x=1}^{x=-1}=\frac{\sum_{k=0}^{6} h_{k} r^{k}}{\sum_{j=0}^{6} \tilde{h}_{k} r^{k}},
$$

where

$$
\begin{aligned}
h_{6}= & -16 b^{4} l^{2} q\left(4 b^{2} l^{2}+b^{2} q^{2}+3\right), \\
h_{5}= & 16 q b^{4} l^{2} m\left(16 b^{2} l^{2}+3 b^{2} q^{2}+9\right), \\
h_{4}= & q\left(192 b^{6} l^{6}-336 b^{6} l^{4} m^{2}-112 b^{6} l^{4} q^{2}\right. \\
& -36 b^{6} l^{2} m^{2} q^{2}-28 b^{6} l^{2} q^{4}-b^{6} q^{6}+112 b^{4} l^{4} \\
& -108 b^{4} l^{2} m^{2}-88 b^{4} l^{2} q^{2}-5 b^{4} q^{4}
\end{aligned}
$$

$$
\begin{aligned}
& \left.+20 b^{2} l^{2}+5 b^{2} q^{2}+1\right), \\
h_{3}= & -4 q b^{2} l^{2} m\left(128 b^{4} l^{4}-36 b^{4} l^{2} m^{2}-80 b^{4} l^{2} q^{2}\right. \\
& \left.-9 b^{4} q^{4}+48 b^{2} l^{2}-30 b^{2} q^{2}+7\right), \\
h_{2}= & -4 q b^{2} l^{2}\left(48 b^{4} l^{6}-80 b^{4} l^{4} m^{2}-68 b^{4} l^{4} q^{2}\right. \\
& +60 b^{4} l^{2} m^{2} q^{2}+20 b^{4} l^{2} q^{4}+3 b^{4} q^{6} \\
& +20 b^{2} l^{4}-8 b^{2} l^{2} m^{2} \\
& \left.-24 b^{2} l^{2} q^{2}+10 b^{2} q^{4}+4 l^{2}-5 q^{2}\right), \\
h_{1}= & q b^{2} l^{4} m\left(64 b^{4} l^{4}+4 b^{4} l^{2} m^{2}-92 b^{4} l^{2} q^{2}\right. \\
& \left.+33 b^{4} q^{4}+12 b^{2} l^{2}-2 b^{2} q^{2}+1\right), \\
h_{0}= & q l^{4}\left(64 b^{6} l^{6}+16 b^{6} l^{4} m^{2}-144 b^{6} l^{4} q^{2}\right. \\
& -12 b^{6} l^{2} m^{2} q^{2}+108 b^{6} l^{2} q^{4}-27 b^{6} q^{6} \\
& +16 b^{4} l^{4}+12 b^{4} l^{2} m^{2}-8 b^{4} l^{2} q^{2} \\
& \left.-3 b^{4} q^{4}-4 b^{2} l^{2}-b^{2} q^{2}-1\right), \\
\tilde{h}_{6}= & 16 b^{4} l^{2}, \\
\tilde{h}_{5}= & -16 b^{3} l(3 b l m+q), \\
\tilde{h}_{4}= & -16 b^{4} l^{4}+36 b^{4} l^{2} m^{2}+24 b^{4} l^{2} q^{2}+b^{4} q^{4} \\
& +24 b^{3} l m q-8 b^{2} l^{2}+6 b^{2} q^{2}+1, \\
\tilde{h}_{3}= & -8 l b\left(4 b^{3} l m q^{2}+b^{2} q^{3}-2 b l m-q\right), \\
\tilde{h}_{2}= & -2 l^{2}\left(8 b^{4} l^{4}-20 b^{4} l^{2} m^{2}\right. \\
& \left.-5 b^{4} q^{4}-16 b^{3} l m q-2 b^{2} q^{2}-1\right), \\
\tilde{h}_{1}= & 8 b l^{3}\left(6 b^{3} l^{3} m-4 b^{3} l m q^{2}\right. \\
& \left.+2 b^{2} l^{2} q-b^{2} q^{3}+2 b l m+q\right), \\
\tilde{h}_{0}= & l^{4}\left(16 b^{4} l^{4}+4 b^{4} l^{2} m^{2}\right. \\
& \left.-24 b^{4} l^{2} q^{2}+9 b^{4} q^{4}+8 b^{3} l m q+8 b^{2} l^{2}-2 b^{2} q^{2}+1\right) .
\end{aligned}
$$

This expression is not what one would have expected from total charge calculation. Even after considering the twosurface $S_{\infty}$ at spacelike infinity, i.e. taking $r \rightarrow \infty$ for the result in (3.3), we obtain $Q_{r \rightarrow \infty}=-q\left(3+b^{2} q^{2}+4 b^{2} l^{2}\right)$ which is finite, but again not as we would expect. However, taking $l \rightarrow 0$ from Eq. (3.3) gives $Q_{l \rightarrow 0}=$ $q\left(1-b^{2} q^{2}\right)$, exactly as the total charge of magnetized Reissner-Nordstrom case [20], without taking the $r \rightarrow \infty$ limit.

Now let us turn the total angular momentum by using the Ernst potential [20],

$J=\frac{|\Lambda(x=1)|^{4}}{8}\left[\Psi+2 A_{\phi} \tilde{A}_{\phi}\right]_{x=1}^{x=-1}$.

It turns out the expression for $J$ above is tedious and we omit to present the full result here. Just like the outcome for $Q$ above, the obtained result for $J$ in (3.4) is still a function of radius $r$. However, considering the two-surface $S_{\infty}$ for $J$ leads to a singular result. Nevertheless, setting the null NUT parameter in the last equation gives the expected result for the magnetized Reissner-Nordstrom spacetime, i.e. $J=$ $-2 b q^{3}\left(1+b^{2} q^{2}\right)$. 


\subsection{Thermodynamics}

Now let us discuss thermodynamical related aspects of the MRNTN spacetime which are vital in establishing the Kerr/CFT correspondence [42] for the black hole in the spacetime. Following the standard formula for black hole area, we can have the area of horizon as

$\mathcal{A}_{H}=\int_{0}^{2 \pi} \mathrm{d} \phi \int_{-1}^{1} \mathrm{~d} x \sqrt{g_{\phi \phi} g_{x x}}=4 \pi\left(r_{+}^{2}+l^{2}\right)$.

This is just the area of a RNTN black hole [31], and consequently the corresponding entropy reads

$S=\frac{\mathcal{A}}{4}=\pi\left(r_{+}^{2}+l^{2}\right)$.

For the Hawking temperature, we can compute by using the complex path method in $(1+1)$ dimension [55]. ${ }^{3}$ Note that the calculation presented in [56] applies to a general stationary and axial symmetric black hole spacetime whose metric takes the form

$d s^{2}=-\tilde{f}(r, x) \mathrm{d} t^{2}+\frac{\mathrm{d} r^{2}}{\tilde{g}(r, x)}+\tilde{C}(r, x) h_{i j}(r, x) \mathrm{d} \tilde{x}^{i} \mathrm{~d} \tilde{x}^{j}$.

In the last equation, we have $\tilde{x}^{i}=[x, \tilde{\phi}]$, and $\tilde{\phi}=\phi-\omega^{\prime} t$, and it is easy to notice that the line element (2.28) can be rewritten in this form. Furthermore, let us consider the geodesic of fixed $x=0$ and $\mathrm{d} \tilde{\phi}=0$. This yields that only the $(t-r)$ sector in the metric which matters. In such consideration, the reading of massless Klein-Gordon equation $\nabla_{\mu} \nabla^{\mu} \Phi$ is simply

$$
\left(\frac{\partial S}{\partial r}\right)^{2}=\frac{1}{\tilde{f}(r) \tilde{g}(r)}\left(\frac{\partial S}{\partial t}\right)^{2},
$$

after employing the Hamilton-Jacobi ansatz for the scalar field $\Phi=\exp [-i S(t, r)]$. Since the spacetime under discussion is stationary, we are allowed to consider

$S(t, r)=E t+\tilde{S}(r)$,

which leads to the solution

$S(t, r)=E\left(t \pm \int_{0}^{r} \frac{\mathrm{d} r}{\sqrt{\tilde{f}(r) \tilde{g}(r)}}\right)$,

for Eq. (3.8). Based on this solution, the ingoing and outgoing fields are

$\Phi_{\text {in }}=\exp \left[-i E\left(t+\int_{0}^{r} \frac{\mathrm{d} r}{\sqrt{\tilde{f}(r) \tilde{g}(r)}}\right)\right]$,

3 The case of Taub-NUT black hole is worked out in [56] and for a Vaidya black hole in [57]. and

$\Phi_{\text {out }}=\exp \left[-i E\left(t-\int_{0}^{r} \frac{\mathrm{d} r}{\sqrt{\tilde{f}(r) \tilde{g}(r)}}\right)\right]$,

respectively. By imposing that the probability of ingoing particle must be unity, i.e. $P_{\text {in }}=\left|\Phi_{\text {in }}\right|^{2}=1$, and by using the detailed balance principle

$P_{\text {out }}=\exp \left(-E / T_{H}\right) P_{\text {in }}$,

finally we can get the Hawking temperature

$T_{H}=\frac{1}{4}\left(\operatorname{Im} \int_{0}^{r} \frac{\mathrm{d} r}{\sqrt{\tilde{f}(r) \tilde{g}(r)}}\right)^{-1}$.

Plugging the metric function (2.28) into the last equation, we have

$T_{H}=\frac{1}{4}\left(\operatorname{Im} \int_{0}^{r} \frac{\left(r^{2}+l^{2}\right) \mathrm{d} r}{\left(r-r_{+}\right)\left(r-r_{-}\right)}\right)^{-1}$,

which then gives us the Hawking temperature for a MRNTN black hole

$T_{H}=\frac{r_{+}-m}{2 \pi\left(r_{+}^{2}+l^{2}\right)}$.

Note that the temperature (3.16) is not a function of external magnetic field parameter $b$, and is exactly the same to that of the generic RNTN black hole [31]. This result for Hawking temperature is in agreement with the one that comes from the standard formula $T_{H}=\kappa / 2 \pi$, where

$\kappa=\sqrt{-\frac{1}{2}\left(\nabla_{\mu} \xi_{\nu}\right)\left(\nabla^{\mu} \xi^{\nu}\right)}$

is the corresponding surface gravity. In the equation above,

$\xi^{\mu} \partial_{\mu}=\partial_{t}-\Omega_{H} \partial \phi$

is the associated Killing vector in the spacetime where the angular velocity at horizon is given by

$\Omega_{H}=-\left.\frac{g_{t \phi}}{g_{\phi \phi}}\right|_{r_{+}}$.

By employing the first law of rotating spacetime with NUT charge proposal in [40], authors of [58] study the Smarr relation and the first law of thermodynamics for the magnetized Kerr-Newman-Taub-NUT spacetime. The MRNTN solution studied in this paper is simply the non-rotating seed limit, i.e. $a=0$, of the general spacetime solution constructed in [58]. Therefore, here we can adopt the Smarr relation presented in [58] for the MRNTN spacetime studied in this paper. It reads 
$\mathcal{M}=\Phi_{H} Q+2 \Omega_{H} \mathcal{J}+\frac{\kappa}{4 \pi} \mathcal{A}_{H}+2 \psi_{+} N_{+}+2 \psi_{-} N_{-}$,

where the total mass $\mathcal{M}$ changes as

$$
\delta \mathcal{M}=\Phi_{H} \delta Q+\Omega_{H} \delta \mathcal{J}+T_{H} \delta S+\psi_{+} \delta N_{+}+\psi_{-} \delta N_{-} .
$$

The total charge $Q$ is given in (3.3), the Coulomb potential is calculated using $\Phi_{H}=-\left.\xi^{\mu} A_{\mu}\right|_{r_{+}}$, the surface gravity $\kappa$ is Eq. (3.17), and horizon area $\mathcal{A}_{H}$ is presented in (3.5). The Misner potentials $\psi_{ \pm}$and Misner charges $N_{ \pm}$for the MRNTN spacetime are given by $[40,58]$

$\psi_{+}=\psi_{-}=\frac{1}{8 \pi l}$,

and

$N_{ \pm} \psi_{ \pm}= \pm \frac{1}{16 \pi} \int_{T_{ \pm}} * \mathrm{~d} \zeta$

accordingly. In equation above, $\zeta$ is the timelike Killing vector, whereas $T_{-}$and $T_{+}$are the Misner string tubes positioned at $x=-1$ and $x=1$, respectively. Furthermore, the total angular momentum $\mathcal{J}=J+J_{+}+J_{-}$in (3.20) gets contributions from the one computed using (3.4), and those related to the Misner strings,

$J_{ \pm}= \pm \frac{1}{16 \pi} \int_{T_{ \pm}} * \mathrm{~d} \chi$

where $\chi$ is the axial Killing vector, i.e. $\chi^{\mu} \partial_{\mu}=\partial_{\phi}$.

Obviously, from (3.20) we understand that the entropy $\mathcal{S}$ of MRNTN black hole is a function of the incorporated charges, namely $\mathcal{S}=\mathcal{S}\left(Q, \mathcal{J}, N_{+}, N_{-}\right)$. Therefore, we can define the chemical potentials [44]

$$
\begin{aligned}
\frac{1}{T_{\phi}} & =\left(\frac{\partial \mathcal{S}_{\mathrm{ext}}}{\partial \mathcal{J}}\right)_{Q, N_{+}, N_{-}}, \frac{1}{T_{q}}=\left(\frac{\partial \mathcal{S}_{\mathrm{ext}}}{\partial Q}\right)_{\mathcal{J}, N_{+}, N_{-}}, \\
\frac{1}{T_{ \pm}} & =\left(\frac{\partial \mathcal{S}_{\mathrm{ext}}}{\partial J}\right)_{\mathcal{J}, Q, N_{\mp}},
\end{aligned}
$$

where $\mathcal{S}_{\text {ext }}$ is the extremal entropy which obeys the balance equation

$\delta \mathcal{S}_{\mathrm{ext}}=\frac{1}{T_{\phi}} \delta \mathcal{J}+\frac{1}{T_{q}} \delta Q+\frac{1}{T_{+}} \delta N_{+}+\frac{1}{T_{-}} \delta N_{-}$.

Since the Hawking temperature $T_{H}$ vanishes at extremality, from Eq. (3.21) we can have

$$
\begin{aligned}
T_{H} \delta \mathcal{S}_{\mathrm{ext}} & =\delta M-\left(\Omega_{H}^{\mathrm{ext}} \delta J+\Phi_{H}^{\mathrm{ext}} \delta Q+\Psi_{+} \delta N_{+}+\Psi_{-} \delta N_{-}\right) \\
& =0 .
\end{aligned}
$$

The thermodynamical relations (3.25), (3.26), and (3.27) that we have established above are later required in obtaining the Frolov-Thorne temperature (4.20) near the extremal MRNTN black hole.

\section{Microscopic entropy for the extremal MRNTN black hole}

In this section, we extend the magnetized Reissner-Nordstrom/ CFT correspondence reported in [37] to the case with the presence of NUT parameter. The first step is to obtain the near horizon geometry for a MRNTN black hole, which can be achieved by performing the transformation

$t \rightarrow \frac{r_{0} t}{\lambda}, \quad r \rightarrow r_{e}+\lambda r_{0} r, \quad \phi \rightarrow \phi+\Omega_{J}^{\mathrm{ext}} \frac{r_{0}}{\lambda} t$.

In equation above $\Omega_{J}=\omega^{\prime}\left(r_{+}\right)$, and $\Omega_{J}^{\mathrm{e} x t}$ is the corresponding quantity evaluated at extremality.

Note that from Eq. (2.6) one can understand that there exist a gauge freedom for the twist potential, namely $\Psi^{\prime} \rightarrow$ $\Psi^{\prime}+\Psi_{0}^{\prime}$ for a constant $\Psi_{0}^{\prime}$, which leaves the Ernst equations to be invariant. Recall that $\Psi^{\prime}$ is the magnetized twist potential satisfying

$-i \nabla \Psi^{\prime}=\frac{f^{\prime 2}}{\rho} \nabla \omega^{\prime}+2 \bar{\Phi}^{\prime} \nabla \Phi^{\prime}$,

where $f^{\prime}, \omega^{\prime}$ are the magnetized metric functions, and $\Phi^{\prime}$ is the magnetized electromagnetic Ernst potential. We then apply the near horizon transformation (4.1) above to the gauged MRNTN metric, with

$\Psi_{0}^{\prime}=\frac{2 m l\left(1+2 b^{2} l^{2}\right)}{b^{2}\left(l^{2}-m^{2}\right)}$.

The resulting near horizon metric of an extremal MRNTN black hole takes the $U(1) \times S L(2, R)$ symmetric form [43],

$$
\begin{aligned}
\mathrm{d} s^{2}= & \Gamma(x)\left\{-r^{2} \mathrm{~d} t^{2}+\frac{\mathrm{d} r^{2}}{r^{2}}+\alpha(x) \mathrm{d} x^{2}\right\} \\
& +\gamma(x)(\mathrm{d} \phi+k r \mathrm{~d} t)^{2},
\end{aligned}
$$

where $r_{0}=q, \alpha(x)=\Delta_{x}^{-1}$,

$\Gamma(x)=\frac{q^{4}\left(4 b^{2}\left(l^{2}-m^{2}\right) x^{2}+q^{2}\left(1+q^{2} b^{2}\right)^{2}\right)}{\left(l^{2}-m^{2}\right)^{2}}$,

$\gamma(x)=\frac{q^{4} \Delta_{x}}{\Gamma(x)}$

and

$k=-\frac{4 q^{3} b\left(1+q^{2} b^{2}\right)}{m^{2}-l^{2}}$.

Moreover, the associated vector field is

$A_{\mu} \mathrm{d} x^{\mu}=L(x)(\mathrm{d} \phi+k r \mathrm{~d} t)$,

where

$L(x)=\frac{q^{4} x \sqrt{q^{2}\left(1+q^{2} b^{2}\right)^{2}-4 b^{2}\left(l^{2}-m^{2}\right)^{2}}}{\Gamma(x)}$. 
One can find that the near horizon geometry (4.4) above possesses the $S L(2, \mathbb{R}) \times U(1)$ isometry. The $S L(2, \mathbb{R})$ symmetry is generated by the Killing vectors

$K_{-}=\partial_{t}$,

$K_{0}=t \partial_{t}-r \partial_{r}$,

$K_{+}=\left(\frac{1}{2 r^{2}}+\frac{t^{2}}{2}\right) \partial_{t}-t r \partial_{r}-\frac{k}{r} \partial_{\phi}$,

obeying $\left[K_{0}, K_{ \pm}\right]= \pm K_{ \pm}$and $\left[K_{-}, K_{+}\right]=K_{0}$, and the $U$ (1) symmetry is generated by $\partial_{\phi}$. Interestingly, the presence of NUT parameter $l$ does not break the $S L(2, \mathbb{R}) \times U(1)$ isometry of the near horizon geometry of a magnetized Reissner-Nordstrom black hole [37]. Furthermore, this isometry hints the compatibility of Kerr/CFT correspondence prescription [42-44] to recover the entropy of extremal black hole in MRNTN spacetime. Moreover, the authors of [43] managed to obtain the central charge for a class of near horizon spacetime and vector field solutions in Einstein-Maxwell theory by using the asymptotic symmetry group (ASG). This work is reviewed and generalized to a class of gravitational theories in [44]. Interestingly, the near horizon metric (4.4) and vector field (4.8) fall into the category discussed in [43], which then allow us to employ the general results presented in [43] to establish the Kerr/CFT correspondence to the charged black hole in MRNTN spacetime.

To ensure that the ASG method can work for the extremal MRNTN black hole, first we need to consider the following boundary condition to the near horizon metric

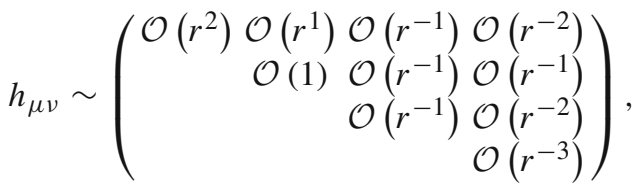

and the following condition for the accompanying vector field

$a_{\mu} \mathrm{d} x^{\mu} \sim \mathcal{O}(r) \mathrm{d} t+\mathcal{O}\left(r^{-1}\right) \mathrm{d} r+\mathcal{O}(1) \mathrm{d} x+\mathcal{O}\left(r^{-2}\right) \mathrm{d} \phi$.

Accordingly, the most general diffeomorphisms preserving the boundary condition for the metric is

$\zeta^{\mu} \partial_{\mu}=\varepsilon(\phi) \partial_{\phi}-r \frac{d \varepsilon(\phi)}{d \phi} \partial_{r}+$ subleading term

which may lead us the associated central charge $[37,43,47]$

$c=c_{\text {grav }}+c_{\text {gauge }}$,

where

$c_{\text {grav }}=3 k \int_{-1}^{+1} d x \sqrt{\Gamma(x) \alpha(x) \gamma(x)}$, and

$c_{\text {gauge }}=0$.

Inserting the metric component (4.4) into Eq. (4.16) gives

$c=-\frac{24 q^{5} b\left(1+q^{2} b^{2}\right)}{q^{2}-2 l^{2}}$.

This result agrees to the central charge associated the near horizon geometry of an extremal magnetized ReissnerNordstrom black hole [37] after taking $b=B / 2$ and $l=0$.

Before we can employ the Cardy formula in recovering the extremal black hole entropy, we need to get the associated near horizon temperature. Clearly the Hawking temperature (3.16) vanishes in extremal limit, which is typical for any other extremal black holes. However, since the Hawking temperature is measured by an observer at infinity, the near horizon temperature is not necessary vanishing in extremal state. Using (3.25)-(3.27), we can have

$T_{\phi}=\lim _{r_{+} \rightarrow m} \frac{T_{H}}{\Omega_{J}^{\mathrm{ext}}-\Omega_{J}}=-\left.\frac{\partial T_{H} / \partial r_{+}}{\partial \Omega_{J} / \partial r_{+}}\right|_{r_{+}=m}$,

that gives us the Frolov-Thorne temperature near an extremal black hole under consideration. For an extremal MRNTN black hole, this temperature reads

$T_{\phi}=\frac{2 l^{2}-q^{2}}{8 \pi q^{3} b\left(1+b^{2} q^{2}\right)}=\frac{1}{2 \pi k}$,

where the constant $k$ is given in (4.7). This result is exactly what we look for so the Kerr/CFT correspondence can provide us the holographic entropy calculation of an extremal MRNTN black hole by using the Cardy formula,

$S_{\text {Cardy }}=\frac{\pi^{2}}{3} c T_{\phi}$

Plugging the central charge (4.19) and Frolov-Thorne temperature (4.21) into the last equation gives us the entropy of an extremal MRNTN black hole,

$S_{\text {ext. }}=\frac{\mathcal{A}_{\text {ext. }}}{4}=\pi q^{2}$.

The fact where Eq. (4.23) is just the extremal limit of Eq. (3.6) allows us to conclude that we have recovered the macroscopic Bekenstein-Hawking entropy for the extremal black hole in MRNTN spacetime by using the microscopic Cardy formula. Obviously, this result supports the conjectured Kerr/CFT correspondence which is considered as an extension of the AdS/CFT correspondence to rotating or charged black holes [42-44]. Similar to the outcomes in previous studies of magnetized black holes [37,47,48], the MRNTN black hole entropy does not get contribution from the external magnetic field. The charge $q$ appearing in (4.23) is simply the conserved electric charge in the seed solution RNTN spacetime (2.19). 


\section{Conclusion}

In this paper, we have presented a new solution in EinsteinMaxwell theory describing the magnetized spacetime outside a charged mass equipped with NUT parameter. The magnetization procedure is performed by using the Ernst prescription, applied to the RNTN solution as the seed. We find that some properties of the MRNTN black hole are just those of the non magnetized one, for example the area of horizon and the Hawking temperature.

Inspired by the Kerr/CFT holography investigations for some magnetized black holes $[37,47,48]$, we extend the Kerr/CFT conjecture to the case of MRNTN case. To proceed, first we need to obtain the corresponding near horizon and accompanying vector solution in an extremal MRNTN geometry. It turns out that resulting near horizon metric and the vector field solution are compatible with the general form that is used in the asymptotic symmetry group method [43]. Therefore, the general formula for the central charge and Frolov-Thorne temperature established in [43] can apply. We pursue this in Sect. 4, where we manage to recover the extremal entropy for a MRNTN black hole by using Cardy formula as prescribed by Kerr/CFT correspondence [42-44].

The spacetime solution presented in this paper is a generalization to the novel solution reported in our previous work, namely the magnetized Taub-NUT spacetime [59]. Obviously, the similar solution generating method should apply if one considers the Kerr-Taub-NUT solution as a seed. Discussing the extremal Kerr/CFT correspondence to the obtained magnetized Kerr-Taub-NUT black hole also worth our consideration, i.e. extending the works in $[47,48]$ to the case with NUT parameter. Further extension of this Kerr/CFT holography to the case of magnetized Kerr-Newman-TaubNUT spacetime is also challenging.

Acknowledgements I thank Merry K. Nainggolan for her encouragement. This work is supported by LPPM UNPAR under contract no III LPPM 2021-02 9-P.

Data Availability Statement This manuscript has associated data in a data repository. [Authors' comment: The paper is posted on arXiv:2101.10302.]

Open Access This article is licensed under a Creative Commons Attribution 4.0 International License, which permits use, sharing, adaptation, distribution and reproduction in any medium or format, as long as you give appropriate credit to the original author(s) and the source, provide a link to the Creative Commons licence, and indicate if changes were made. The images or other third party material in this article are included in the article's Creative Commons licence, unless indicated otherwise in a credit line to the material. If material is not included in the article's Creative Commons licence and your intended use is not permitted by statutory regulation or exceeds the permitted use, you will need to obtain permission directly from the copyright holder. To view a copy of this licence, visit http://creativecomm ons.org/licenses/by/4.0/.

Funded by SCOAP ${ }^{3}$.
Apprndix A: Near horizon geometry and the twist potential

Note that one can "gauge" the twist potential $\Psi$ in (2.4) by adding some constant $\Psi_{0}$ to it. It results some changes in the magnetized solutions, and in particular for the metric functions $f^{\prime} \rightarrow f^{\prime}+\Delta f^{\prime}$ and $\omega^{\prime} \rightarrow \omega^{\prime}+\Delta \omega^{\prime}$. These changes are

$$
\begin{aligned}
\Delta \omega^{\prime}= & -\frac{2 b^{3} \Psi_{0}}{\Delta_{x} r^{4}+2 l^{2}\left(1-3 x^{2}\right) r^{2}+l^{2}\left(l^{2}+3 l^{2} x^{2}-4 q^{2} x^{2}\right)} \\
& \times\left\{2 b \Delta_{r}\left(2 l^{2} m-3 r l^{2}-r^{3}\right) x^{3}+2 l q\left(2 q^{2}+3 r^{2}\right.\right. \\
& \left.\left.-4 m r-l^{2}\right) x^{2}+b\left(6 r l^{2}-l \Psi_{0}+2 r^{3}\right) x-2 q l\left(r^{2}+l^{2}\right)\right\},
\end{aligned}
$$

and

$$
\Delta f^{\prime}=-b^{3} \Psi_{0} \frac{\Xi_{1} \Xi_{2}}{\Xi_{3} \Xi_{4}},
$$

where

$$
\begin{aligned}
\Xi_{1}= & \Delta_{x} r^{4}+\left(2 l^{2}-6 l^{2} x^{2}\right) r^{2}+8 m r l^{2} x^{2} \\
& -4 q^{2} l^{2} x^{2}+3 l^{4} x^{2}+l^{4}, \\
\Xi_{2}= & 4 b l\left(3 r l^{2}-l^{2} m-r^{3}-2 q^{2} r+3 m r^{2}\right) x^{2}+4 q\left(l^{2}-r^{2}\right) x \\
& +b\left(r^{2}+l^{2}\right)\left(\Psi_{0}-4 r l\right), \\
\Xi_{3}= & \Delta_{x}^{2} b^{4} r^{6}+b^{2}\left(2 b^{2} x^{2} q^{2}+7 l^{2} b^{2}-6 b^{2} l^{2} x^{2}+15 b^{2} l^{2} x^{4}\right. \\
& \left.-2 b^{2} q^{2} x^{4}-2 x^{2}+2\right) r^{4}-4 l b^{3}\left(2 m l x^{2} b+10 b l x^{4} m\right. \\
& \left.+\Psi_{0} x^{2} b+\Psi_{0} b-4 x^{3} q\right) r^{3}+\left(12 l^{2} b^{4} x^{2} q^{2}+4 l^{2} b^{2}\right. \\
& +12 l^{2} b^{4} x^{4} q^{2}-24 l b^{3} m x^{3} q+b^{4} x^{4} q^{4}+12 l b^{4} m x^{2} \Psi_{0} \\
& -12 b^{2} l^{2} x^{2}+7 b^{4} l^{4}+\Psi_{0}^{2} b^{4}+6 b^{2} x^{2} q^{2} \\
& \left.-9 l^{4} b^{4} x^{4}-4 b^{3} q x C+1-30 l^{4} b^{4} x^{2}+36 l^{2} b^{4} m^{2} x^{4}\right) r^{2} \\
& +4 b l\left(2 l b^{3} m\left\{3 l^{2}-4 q^{2}\right\} x^{4}+2 b^{2} x^{3} q^{3}\right. \\
& +\left\{4 l m b+3 b^{3} \Psi_{0} l^{2}+6 l^{3} b^{3} m-2 b^{3} q^{2} \Psi_{0}\right\} x^{2} \\
& \left.-\left\{2 q+4 l^{2} q b^{2}\right\} x-b^{3} \Psi_{0} l^{2}\right) r+l^{2} b^{4} \Psi_{0}^{2} \\
& +\left(4 l^{2} b^{3} q x-4 l^{3} b^{4} m x^{2}\right) \Psi_{0}+l^{2} \\
& -18 l^{4} b^{4} x^{4} q^{2}-2 b^{2} l^{2} x^{2} q^{2}+6 l^{6} b^{4} x^{2} \\
& +2 b^{2} l^{4}+6 b^{2} l^{4} x^{2}+9 l^{2} b^{4} x^{4} q^{4} \\
& +4 l^{4} b^{4} m^{2} x^{4}-8 l^{3} b^{3} q x^{3} m+l^{6} b^{4} \\
& +9 l^{6} b^{4} x^{4}-6 l^{4} b^{4} x^{2} q^{2}, \\
\Xi_{4}= & \Xi_{3}-b^{3} \Psi_{0}\left\{4 b l\left(3 r l^{2}-l^{2} m-r^{3}-2 q^{2} r+3 m r^{2}\right) x^{2}\right. \\
& \left.+4 q\left(r^{2}-l^{2}\right) x+b\left(r^{2}+l^{2}\right)\left(\Psi_{0}-4 r l\right)\right\} .
\end{aligned}
$$

\section{Appendix B: Kretschmann scalar for MRNTN spacetime at $x=0$}

The Kretschmann scalar for MRNTN spacetime evaluated at equator, i.e. $x=0$, can be expressed as 
$K_{x=0}=\frac{\sum_{j=0}^{12} k_{2 j} l^{2 j}}{\left(l^{2}+r^{2}\right)^{6}\left(b^{4} l^{4}+6 b^{4} l^{2} r^{2}+b^{4} r^{4}+2 b^{2} l^{2}+2 b^{2} r^{2}+1\right)^{6}}$,

where

$$
\begin{aligned}
& k_{0}=8 r^{4}\left(b^{2} r^{2}+1\right)^{4}\left(126 b^{8} m^{2} r^{10}\right. \\
& -144 b^{8} m q^{2} r^{9}-108 b^{8} m r^{11} \\
& +43 r^{8} q^{4} b^{8}+60 r^{10} q^{2} b^{8}+24 b^{8} r^{12}+86 r^{4} q^{4} b^{4} \\
& -216 b^{6} m^{2} r^{8}+676 b^{6} m q^{2} r^{7} \\
& +204 b^{6} m r^{9}-264 r^{6} q^{4} b^{6} \\
& -356 r^{8} q^{2} b^{6}-48 b^{6} r^{10}+124 b^{4} m^{2} r^{6} \\
& -36 b^{4} m q^{2} r^{5}-148 b^{4} m r^{7} \\
& +36 b^{4} q^{2} r^{6}+40 b^{4} r^{8}+24 b^{2} m^{2} r^{4} \\
& +28 b^{2} m q^{2} r^{3}-12 b^{2} m r^{5} \\
& \left.-48 r^{2} q^{4} b^{2}+4 b^{2} q^{2} r^{4}+6 m^{2} r^{2}-12 m q^{2} r+7 q^{4}\right), \\
& k_{2}=-16 r^{2}\left(b^{2} r^{2}+1\right)^{2}\left(261 b^{12} m^{2} r^{14}\right. \\
& -204 b^{12} m q^{2} r^{13}-390 b^{12} m r^{15} \\
& +17 b^{12} q^{4} r^{12}+186 b^{12} q^{2} r^{14} \\
& -1230 b^{10} m^{2} r^{12}-2034 b^{10} m q^{2} r^{11}+1152 b^{10} m r^{13} \\
& +1148 b^{10} q^{4} r^{10}+1166 b^{10} q^{2} r^{12} \\
& -276 b^{10} r^{14}+1963 b^{8} m^{2} r^{10} \\
& -7844 b^{8} m q^{2} r^{9}-1608 b^{8} m r^{11} \\
& +2367 r^{8} q^{4} b^{8}+3770 r^{10} q^{2} b^{8} \\
& +353 b^{8} r^{12}-972 b^{6} m^{2} r^{8}+3124 b^{6} m q^{2} r^{7}+1184 b^{6} m r^{9} \\
& -1792 r^{6} q^{4} b^{6}-1972 r^{8} q^{2} b^{6}-304 b^{6} r^{10}+67 b^{4} m^{2} r^{6} \\
& +780 b^{4} m q^{2} r^{5}-174 b^{4} m r^{7}+1007 r^{4} q^{4} b^{4} \\
& -482 b^{4} q^{2} r^{6}+71 b^{4} r^{8} \\
& +286 b^{2} m q^{2} r^{3}-192 b^{2} m r^{5}-300 r^{2} q^{4} b^{2} \\
& -42 b^{2} q^{2} r^{4}+36 b^{2} r^{6}+45 m^{2} r^{2}-60 m q^{2} r-36 m r^{3} \\
& \left.+117 b^{12} r^{16}+186 b^{2} m^{2} r^{4}+17 q^{4}+30 q^{2} r^{2}+3 r^{4}\right) \text {, } \\
& k_{4}=\left(76992 m q^{2} r-74544 m^{2} r^{2}+68832 m r^{3}\right. \\
& \left.-18056 q^{4}-35616 q^{2} r^{2}-15120 r^{4}\right) r^{16} b^{16} \\
& +\left(43584 m^{2} r^{2}+304128 m q^{2} r-24096 m r^{3}\right. \\
& \left.-139008 q^{4}-162048 q^{2} r^{2}+1536 r^{4}\right) r^{14} b^{14} \\
& +\left(25216 m^{2} r^{2}+860736 m q^{2} r-43136 m r^{3}\right. \\
& \left.-292864 q^{4}-401216 q^{2} r^{2}+7520 r^{4}\right) r^{12} b^{12} \\
& +\left(142240 m q^{2} r-156992 m^{2} r^{2}+58752 m r^{3}+52896 q^{4}\right. \\
& \left.+23776 q^{2} r^{2}-8384 r^{4}\right) r^{10} b^{10} \\
& +\left(11840 r^{4}-13472 m^{2} r^{2}-821024 m q^{2} r-46752 m r^{3}\right. \\
& \left.+134992 q^{4}+434464 q^{2} r^{2}\right) r^{8} b^{8} \\
& +\left(12512 m r^{3}-28480 m^{2} r^{2}-230080 m q^{2} r\right. \\
& \left.-67008 q^{4}+67264 q^{2} r^{2}-2944 r^{4}\right) r^{6} b^{6} \\
& +\left(3232 r^{4}-23424 m^{2} r^{2}+2048 m q^{2} r\right. \\
& \left.+6080 m r^{3}+72960 q^{4}-23168 q^{2} r^{2}\right) r^{4} b^{4} \\
& +\left(3648 r^{4}-960 m^{2} r^{2}+16672 m q^{2} r-6720 m r^{3}\right. \\
& \left.-7136 q^{4}-10912 q^{2} r^{2}\right) r^{2} b^{2} \\
& +720 m^{2} r^{2}-480 m q^{2} r-1920 m r^{3}+56 q^{4} \\
& +960 q^{2} r^{2}+720 r^{4} \text {, }
\end{aligned}
$$

$$
\begin{aligned}
& k_{6}=-282096 b^{16} m^{2} r^{16}+302592 b^{16} m q^{2} r^{15} \\
& +184608 b^{16} m r^{17} \\
& -75968 b^{16} q^{4} r^{14}-87840 b^{16} q^{2} r^{16}-30576 b^{16} r^{18} \\
& +328704 b^{14} m^{2} r^{14}+788224 b^{14} m q^{2} r^{13} \\
& -240384 b^{14} m r^{15} \\
& -361472 b^{14} q^{4} r^{12}-386816 b^{14} q^{2} r^{14}+26592 b^{14} r^{16} \\
& +111744 b^{12} m^{2} r^{12}+550464 b^{12} m q^{2} r^{11} \\
& -159104 b^{12} m r^{13}+48768 b^{12} q^{4} r^{10} \\
& -116544 b^{12} q^{2} r^{12}+3072 b^{12} r^{14} \\
& -247488 b^{10} m^{2} r^{10}-2836512 b^{10} m q^{2} r^{9} \\
& -102656 b^{10} m r^{11}+859872 b^{10} q^{4} r^{8} \\
& +1431968 b^{10} q^{2} r^{10}+24896 b^{10} r^{12} \\
& +106656 b^{8} m^{2} r^{8}-1326080 b^{8} m q^{2} r^{7}-253472 b^{8} m r^{9} \\
& -185408 b^{8} q^{4} r^{6}+241920 b^{8} q^{2} r^{8}+40448 b^{8} r^{10} \\
& +26240 b^{6} m^{2} r^{6}+133440 b^{6} m q^{2} r^{5}-104832 b^{6} m r^{7} \\
& +433984 b^{6} q^{4} r^{4}-209984 b^{6} q^{2} r^{6}+25952 b^{6} r^{8} \\
& +15104 b^{4} m^{2} r^{4}+78528 b^{4} m q^{2} r^{3} \\
& -54464 b^{4} m r^{5}-58496 b^{4} q^{4} r^{2} \\
& -49472 b^{4} q^{2} r^{4}+15424 b^{4} r^{6} \\
& +3648 b^{2} m^{2} r^{2}-5408 b^{2} m q^{2} r-8064 b^{2} m r^{3} \\
& +736 b^{2} q^{4}+12960 b^{2} q^{2} r^{2}+384 b^{2} r^{4} \\
& -48 m^{2}+576 m r-96 q^{2}-720 r^{2} \text {, } \\
& k_{8}=48+\left(268032 m q^{2} r^{13}-277920 m^{2} r^{14}-14784 m r^{15}\right. \\
& \left.-57040 q^{4} r^{12}+81600 q^{2} r^{14}+10512 r^{16}\right) b^{16} \\
& +\left(1483776 m^{2} r^{12}-400064 m q^{2} r^{11}-862272 m r^{13}\right. \\
& \left.+167488 q^{4} r^{10}+39360 q^{2} r^{12}+32256 r^{14}\right) b^{14} \\
& +\left(431488 m^{2} r^{10}-4368128 m q^{2} r^{9}-336448 m r^{11}\right. \\
& \left.+1931392 q^{4} r^{8}+1885312 q^{2} r^{10}-73600 r^{12}\right) b^{12} \\
& +\left(353472 m^{2} r^{8}-3844768 m q^{2} r^{7}-778176 m r^{9}\right. \\
& \left.+147296 q^{4} r^{6}+474656 q^{2} r^{8}+66112 r^{10}\right) b^{10} \\
& +\left(365344 m^{2} r^{6}+363296 m q^{2} r^{5}-356704 m r^{7}\right. \\
& \left.+1202000 q^{4} r^{4}-846368 q^{2} r^{6}-38080 r^{8}\right) b^{8} \\
& +\left(51328 m^{2} r^{4}+158304 m q^{2} r^{3}-50336 m r^{5}\right. \\
& \left.-224416 q^{4} r^{2}-25056 q^{2} r^{4}-35200 r^{6}\right) b^{6} \\
& +\left(3968 m^{2} r^{2}-17952 m q^{2} r+4704 m r^{3}\right. \\
& \left.+3584 q^{4}+51936 q^{2} r^{2}-19712 r^{4}\right) b^{4} \\
& +\left(2976 m r-192 m^{2}-1024 q^{2}-3648 r^{2}\right) b^{2}, \\
& k_{10}=32 b^{2}\left(25599 b^{14} m^{2} r^{12}-34644 b^{14} m q^{2} r^{11}\right. \\
& -24930 b^{14} m r^{13}+11277 b^{14} q^{4} r^{10}+20238 b^{14} q^{2} r^{12} \\
& +1071 b^{14} r^{14}+88212 b^{12} m^{2} r^{10}-138726 b^{12} m q^{2} r^{9} \\
& -12444 b^{12} m r^{11}+61434 b^{12} q^{4} r^{8}+25294 b^{12} q^{2} r^{10} \\
& -11670 b^{12} r^{12}+21840 b^{10} m^{2} r^{8}-163042 b^{10} m q^{2} r^{7} \\
& +2714 b^{10} m r^{9}+36932 b^{10} q^{4} r^{6} \\
& -18 b^{10} q^{2} r^{8}-10966 b^{10} r^{10} \\
& +15494 b^{8} m^{2} r^{6}-7751 b^{8} m q^{2} r^{5} \\
& -2072 b^{8} m r^{7}+54377 b^{8} q^{4} r^{4} \\
& -52197 b^{8} q^{2} r^{6}-11380 b^{8} r^{8}+2327 b^{6} m^{2} r^{4} \\
& +7240 b^{6} m q^{2} r^{3}+10161 b^{6} m r^{5}
\end{aligned}
$$




$$
\begin{aligned}
& -14855 b^{6} q^{4} r^{2}+3912 b^{6} q^{2} r^{4} \\
& -8180 b^{6} r^{6}-314 b^{4} m^{2} r^{2}-693 b^{4} m q^{2} r \\
& +2254 b^{4} m r^{3}+287 b^{4} q^{4}+3057 b^{4} q^{2} r^{2}-1667 b^{4} r^{4} \\
& \left.-6 b^{2} m^{2}+157 b^{2} m r-114 b^{2} q^{2}-121 b^{2} r^{2}+9\right) \text {, } \\
& k_{12}=16 b^{4}\left(131850 b^{12} m^{2} r^{10}-175368 b^{12} m q^{2} r^{9}\right. \\
& -19044 b^{12} m r^{11} \\
& +56531 b^{12} q^{4} r^{8}+26364 b^{12} q^{2} r^{10} \\
& -23898 b^{12} r^{12}+91464 b^{10} m^{2} r^{8} \\
& -223280 b^{10} m q^{2} r^{7}+174924 b^{10} m r^{9} \\
& +91072 b^{10} q^{4} r^{6}-102512 b^{10} q^{2} r^{8} \\
& -61008 b^{10} r^{10}-13640 b^{8} m^{2} r^{6} \\
& -94884 b^{8} m q^{2} r^{5}+95672 b^{8} m r^{7} \\
& +83840 b^{8} q^{4} r^{4}-108460 b^{8} q^{2} r^{6}-33652 b^{8} r^{8} \\
& +1156 b^{6} m^{2} r^{4}+27486 b^{6} m q^{2} r^{3} \\
& +42360 b^{6} m r^{5}-37042 b^{6} q^{4} r^{2} \\
& -9734 b^{6} q^{2} r^{4}-18916 b^{6} r^{6}-1566 b^{4} m^{2} r^{2} \\
& -262 b^{4} m q^{2} r+9970 b^{4} m r^{3}+875 b^{4} q^{4} \\
& +9414 b^{4} q^{2} r^{2}+92 b^{4} r^{4}+12 b^{2} m^{2} \\
& \left.-94 b^{2} m r-432 b^{2} q^{2}+792 b^{2} r^{2}+50\right), \\
& k_{14}=32 b^{6}\left(29529 b^{10} m^{2} r^{8}-46272 b^{10} m q^{2} r^{7}+74898 b^{10} m r^{9}\right. \\
& +17114 b^{10} q^{4} r^{6}-41874 b^{10} q^{2} r^{8}-23103 b^{10} r^{10} \\
& -13584 b^{8} m^{2} r^{6}-36336 b^{8} m q^{2} r^{5} \\
& +91176 b^{8} m r^{7}+15912 b^{8} q^{4} r^{4} \\
& -50672 b^{8} q^{2} r^{6}+3894 b^{8} r^{8}-4964 b^{6} m^{2} r^{4} \\
& +19974 b^{6} m q^{2} r^{3}+8308 b^{6} m r^{5}-13588 b^{6} q^{4} r^{2} \\
& -26766 b^{6} q^{2} r^{4}+11496 b^{6} r^{6}-242 b^{4} m^{2} r^{2} \\
& +261 b^{4} m q^{2} r+3304 b^{4} m r^{3} \\
& +413 b^{4} q^{4}+8511 b^{4} q^{2} r^{2}+4550 b^{4} r^{4} \\
& +15 b^{2} m^{2}-411 b^{2} m r \\
& \left.-280 b^{2} q^{2}+1448 b^{2} r^{2}+25\right), \\
& k_{16}=8 b^{8}\left(534 b^{8} m^{2} r^{6}-11280 b^{8} m q^{2} r^{5}\right. \\
& +195300 b^{8} m r^{7}+8879 b^{8} q^{4} r^{4} \\
& -140052 b^{8} q^{2} r^{6}+56124 b^{8} r^{8} \\
& -30048 b^{6} m^{2} r^{4}+57044 b^{6} m q^{2} r^{3} \\
& +5484 b^{6} m r^{5}-21772 b^{6} q^{4} r^{2} \\
& -98596 b^{6} q^{2} r^{4}+126480 b^{6} r^{6} \\
& +2944 b^{4} m^{2} r^{2}-300 b^{4} m q^{2} r \\
& -21036 b^{4} m r^{3}+952 b^{4} q^{4}+44724 b^{4} q^{2} r^{2}+26392 b^{4} r^{4} \\
& \left.+24 b^{2} m^{2}-1124 b^{2} m r-1152 b^{2} q^{2}+6072 b^{2} r^{2}-48\right) \text {, } \\
& k_{18}=-16 b^{10}\left(6345 b^{6} m^{2} r^{4}-7548 b^{6} m q^{2} r^{3}\right. \\
& -10782 b^{6} m r^{5}+1841 b^{6} q^{4} r^{2} \\
& +12450 b^{6} q^{2} r^{4}-35847 b^{6} r^{6} \\
& -1524 b^{4} m^{2} r^{2}+590 b^{4} m q^{2} r+19188 b^{4} m r^{3} \\
& -154 b^{4} q^{4}-15030 b^{4} q^{2} r^{2}-13794 b^{4} r^{4} \\
& \left.+12 b^{2} m^{2}-494 b^{2} m r+444 b^{2} q^{2}+1334 b^{2} r^{2}+58\right), \\
& k_{20}=8 b^{12}\left(882 b^{4} m^{2} r^{2}\right. \\
& -456 b^{4} m q^{2} r-16404 b^{4} m r^{3}+43 b^{4} q^{4}+7788 b^{4} q^{2} r^{2} \\
& +16758 b^{4} r^{4}-24 b^{2} m^{2}+1452 b^{2} m r-416 b^{2} q^{2}
\end{aligned}
$$

\section{References}

1. J.B. Griffiths, J. Podolsky, Exact Space-Times in Einstein's General Relativity (Cambridge University Press, 2009). https://doi.org/10. 1017/CBO9780511635397

2. W.B. Feng, S.J. Yang, Q. Tan, J. Yang, Y.X. Liu, Sci. China Phys. Mech. Astron. 64(6), 260411 (2021)

3. X.Y. Wang, J. Jiang, JHEP 05, 161 (2020)

4. Y.W. Han, K.J. He, Y. Hong, Int. J. Theor. Phys. 59(5), 1537-1546 (2020)

5. Y. Mo, Y. Tian, B. Wang, H. Zhang, Z. Zhong, Phys. Rev. D 98(12), 124025 (2018)

6. M.R. Garousi, A. Ghodsi, Phys. Lett. B 687, 79-83 (2010)

7. C.M. Chen, Y.M. Huang, S.J. Zou, JHEP 03, 123 (2010)

8. C.M. Chen, Y.M. Huang, J.R. Sun, M.F. Wu, S.J. Zou, Phys. Rev. D 82, 066003 (2010)

9. R.M. Wald, Phys. Rev. D 10, 1680 (1974)

10. F.J. Ernst, J. Math. Phys. 17, 54 (1976)

11. M.A. Melvin, Phys. Lett. 8, 65 (1964)

12. W.J. Wild, R.M. Kerns, Phys. Rev. D 21, 332-335 (1980)

13. F.J. Ernst, W.J. Wild, J. Math. Phys. 17, 182 (1976)

14. A.N. Aliev, D.V. Galtsov, Sov. Phys. Usp. 32, 75 (1989)

15. R. Brito, V. Cardoso, P. Pani, Phys. Rev. D 89(10), 104045 (2014)

16. J. Bicak, F. Hejda, Phys. Rev. D 92(10), 104006 (2015)

17. M. Kološ, Z. Stuchlik, A. Tursunov, Class. Quantum Gravity 32(16), 165009 (2015)

18. A. Tursunov, M. Kolos, Z. Stuchlik, B. Ahmedov, Phys. Rev. D 90(8), 085009 (2014)

19. M. Astorino, G. Compère, R. Oliveri, N. Vandevoorde, Phys. Rev. D 94(2), 024019 (2016)

20. I. Booth, M. Hunt, A. Palomo-Lozano, H.K. Kunduri, Class. Quantum Gravity 32(23), 235025 (2015)

21. G.W. Gibbons, A.H. Mujtaba, C.N. Pope, Class. Quantum Gravity 30(12), 125008 (2013)

22. G.W. Gibbons, Y. Pang, C.N. Pope, Phys. Rev. D 89(4), 044029 (2014)

23. A.N. Aliev, D.V. Galtsov, Astrophys. Space Sci. 155, 181 (1989)

24. A.N. Aliev, D.V. Galtsov, A.A. Sokolov, Sov. Phys. J. 23, 179 (1980)

25. A.N. Aliev, D.V. Galtsov, V.I. Petrukhov, Astrophys. Space Sci. 124, 137 (1986)

26. A.N. Aliev, D.V. Galtsov, Sov. Phys. JETP 67, 1525 (1988)

27. A.N. Aliev, D.V. Galtsov, Sov. Phys. J. 32, 790 (1989)

28. D.V. Galtsov, V.I. Petukhov, Zh. Eksp, Teor. Fiz. 74, 801 (1978)

29. W.A. Hiscock, J. Math. Phys. 22, 1828 (1981)

30. H. Cebeci, N. Özdemir, S. Şentorun, Phys. Rev. D 93(10), 104031 (2016)

31. P. Pradhan, Mod. Phys. Lett. A 30(35), 1550170 (2015)

32. D. Bini, C. Cherubini, R.T. Jantzen, B. Mashhoon, Class. Quantum Gravity 20, 457-468 (2003)

33. A. Cisterna, A. Neira-Gallegos, J. Oliva, S.C. Phys. Rev. D 103(6), 064050 (2021)

34. H.M. Siahaan, Phys. Rev. D 102(6), 064022 (2020)

35. H.M. Siahaan, Eur. Phys. J. C 80(10), 1000 (2020)

36. L. Ciambelli, C. Corral, J. Figueroa, G. Giribet, R. Olea, Phys. Rev. D 103(2), 024052 (2021) 
37. M. Astorino, JHEP 1510, 016 (2015)

38. R.A. Hennigar, D. Kubizńák, R.B. Mann, Phys. Rev. D 100(6), 064055 (2019)

39. A.B. Bordo, F. Gray, D. Kubizňák, JHEP 07, 119 (2019)

40. A. Ballon Bordo, F. Gray, R.A. Hennigar, D. Kubizňák, Phys. Lett. B 798, 134972 (2019)

41. V.P. Frolov, P. Krtous, D. Kubiznak, Phys. Lett. B 771, 254-256 (2017)

42. M. Guica, T. Hartman, W. Song, A. Strominger, Phys. Rev. D 80, 124008 (2009)

43. T. Hartman, K. Murata, T. Nishioka, A. Strominger, JHEP 04, 019 (2009)

44. G. Compère, Living Rev. Relativ. 15, 11 (2012)

45. A.M. Ghezelbash, Mod. Phys. Lett. A 27, 1250046 (2012)

46. M.F.A.R. Sakti, A. Suroso, F.P. Zen, Int. J. Mod. Phys. D 27(12), 1850109 (2018)
47. H.M. Siahaan, Class. Quantum Gravity 33(15), 155013 (2016)

48. M. Astorino, Phys. Lett. B 751, 96 (2015)

49. M. Hossain Ali, K. Sultana, Int. J. Theor. Phys. 52, 2802-2817 (2013)

50. M.H. Ali, K. Sultana, Int. J. Theor. Phys. 52, 4537-4556 (2013)

51. S.Q. Wu, D. Wu, Phys. Rev. D 100(10), 101501 (2019)

52. G. Clément, D. Gal'tsov, Phys. Lett. B 802, 135270 (2020)

53. G. Clément, M. Guenouche, Gen. Relativ. Gravit. 50(6), 60 (2018)

54. B.K. Harrison, J. Math. Phys. 9, 1744 (1968)

55. K. Srinivasan, T. Padmanabhan, Phys. Rev. D 60, 024007 (1999)

56. R. Kerner, R.B. Mann, Phys. Rev. D 73, 104010 (2006)

57. H.M. Siahaan, Triyanta, Int. J. Mod. Phys. A 25, 145-153 (2010)

58. M. Ghezelbash, H.M. Siahaan, Eur. Phys. J. C 81(7), 621 (2021)

59. H.M. Siahaan, Magnetized Taub-NUT spacetime. arXiv:2101.06510 [gr-qc] 